\title{
Clinical Profile of US Combat Veterans with PTSD on M-FAST Items
}

\author{
Zack Z. Cernovsky, Stephan C. Mann, and Varadaraj R. Velamoor
}

\section{ABSTRACT}

Background: The Miller Forensic Assessment of Symptoms Test (MFAST; Miller, 2001) is a widely used but controversial instrument promulgated to measure malingering. Its use is encouraged by publications which are methodologically flawed such as the recent metaanalysis by Detullio et al. (2019). In this study, we examine which of MFAST's 25 items are most frequently endorsed by veterans with posttraumatic stress disorder (PTSD).

Method: Using tabular data published by Wolf's team in 2020, we identified from a clinical perspective, the 7 M-FAST items endorsed by the highest proportions of 121 US combat veterans with a probable current diagnosis of PTSD. Since the M-FAST diagnostic cutoff is 6 or more points and each endorsed item counts as one point, the 7 most frequently endorsed M-FAST items provide a clinical profile, via their content, as to which items are most frequently involved in misdiagnosing veterans as malingerers.

Results and Discussion: Item 2 ("feeling depressed most of the time") was endorsed by $67.8 \%$, Item 23 ("feeling that I don't really matter") by $56.2 \%$, Item 20 (formication) by $28.9 \%$, and Item 17 (phantosmia, i.e., "false sensation of an odor") by $24.0 \%$. Formication and phantosmia are legitimate neuropsychological symptoms that may occur with whiplash injuries and head trauma or exposure to toxic chemicals in combat. Item 21 ("at times hearing music coming from nowhere") endorsed by $22.3 \%$ may describe spontaneous intrapsychic activity of musically inclined persons. Item 14 ("Sometimes it seems as if someone controls my symptoms, turning them on and off") endorsed by $20.7 \%$ may reflect the unpredictable fluctuation of frequency and severity of PTSD symptoms. Item 1 ("restlessness while seated") endorsed by $19.8 \%$ is scored in the MFAST as a discrepancy between the patient reporting that he "often" feels restless, and the examiner's observation that no such nonverbal behavior during the brief M-FAST interview was noted. Restlessness is a postconcussive symptom that is not necessarily always present, such as during the M-FAST interview.

Conclusions: All 7 most frequently endorsed items by combat veterans are neither exclusive to malingerers nor pathognomonic of malingering. They form a pattern of legitimate medical symptoms fallaciously scored and interpreted in the M-FAST's system as indicators of malingering.

Keywords: malingering, M-FAST, veterans, PTSD, post-concussion, whiplash.

\section{INTRODUCTION}

The Miller Forensic Assessment of Symptoms Test (MFAST) [1] was developed by Holly A. Miller to identify malingering of psychiatric symptoms. The M-FAST was originally intended for use in forensic patients, but has subsequently been widely extended to other clinical populations without being accurately validated for such "off label use." For example, the M-FAST has been used in the
Published Online: July 21, 2021

ISSN: $2736-5476$

DOI: 10.24018/ejclinicmed.2021.2.3.53

\section{Z. Z. Cernovsky*}

Dept. of Psychiatry, Western University,

London, Ontario, Canada.

(e-mail: zcernovs@uwo.ca)

S. C. Mann*

Central Montgomery Behavioral Heath,

Norristown, PA, USA.

(e-mail: smann1234@aol.com)

V. R. Velamoor

Dept. of Psychiatry, Laurentian and

Lakehead Universities, and Western

University, Ontario, Canada.

(e-mail: velamoorraj@gmail.com)

*Corresponding Authors assessment of injured motorists engaged in insurance litigations, or on patients of the US Department of Veterans Affairs (VA) applying for PTSD compensation benefits. The test development standards specified by the American Psychological Association require that a new test be separately validated for each particular clinical group on which it is to be used [2].

Psychometric performance of the M-FAST on a sample of US combat veterans was evaluated by a group of VA 
investigators led by Erika Wolf [3]. After the veterans completed the 25 item M-FAST, they then underwent a "testing-the-limits" follow-up interview in which a clinician explored with them in detail each item that they had endorsed. All interviews were videotaped. Following this additional probing and clarification, if the participant still endorsed the item, the research team determined if the item should be scored as malingering.

The "testing-the-limits" interviews showed that, on all 25 items, without exception, the M-FAST overestimated the likelihood of malingering in a manner that was dissonant with clinical impressions of Wolf's team[3,4]. For instance, MFAST Item 2 ("feeling depressed") was endorsed initially by $67.8 \%$ of veterans with PTSD, but then subsequent interviews with clinicians determined that their response to Item 2 would qualify as malingering in only $5.7 \%$ of these veterans [3].

Another large overestimate of malingering was noted with Item 23 ("I don't matter"), endorsed in the standard administration of the M-FAST by $56.2 \%$ of veterans with PTSD, but then the "testing-the-limits" interviews by VA clinicians determined that only $3.8 \%$ of their responses to this item could be considered indicative of malingering [3].

The magnitude of these overestimates of malingering on all 25 M-FAST items corresponded to a point biserial correlation coefficient $\mathrm{r}=0.49$ ( $\mathrm{p}<0.001$, 2-tailed) or Cohen's $\mathrm{d}=1.12[5]$.

Too many M-FAST items are semantically ambiguous. For instance, Items 15, 18, and 22 refer to "hearing voices" without making it sufficiently clear if the item means hallucinations of voices or a regular auditory perception of others talking (i.e., no hallucination). For instance, Item 15 "When I hear voices, I hear them from either my right or my left ear ...." could be misunderstood as querying for disorders of hearing, which are not uncommon in veterans after repeated exposure to explosive blasts.

Misinterpretation of such items raises the likelihood of these patients being classified as malingerers. The instructions provided in the M-FAST manual to staff administering the test convey impatience and lack of empathy, as they urge the examiner to avoid explanations of items to patients, if the patients request clarifications, see MFAST manual [1], page 10: "Some examinees will typically respond to interview items with questions of their own. With few exceptions, the interviewer should respond by saying 'I am interested in your perceptions, ' and then repeat the item." And on the same page of M-FAST manual [1]: "It is very important for the interviewer to correctly set the stage from the beginning of the administration. Allowing the examinee to provide no response or to elaborate extensively on his or her symptoms will likely result in a difficult, time-consuming, and non-standardized administration. Interviewers may believe that it is impolite to interrupt when an examinee begins to elaborate on his or her symptoms or psychological problems during the interview. However, the interviewer must be able to redirect the examinee to respond to each item with minimal or no elaboration."

Some M-FAST items have 2 parts, similar to this fictitious example:

"I have terrifying nightmares." - "I have them only on days other than Monday." The second part of the item alters or contradicts the meaning of its first part: this leaves some patients puzzled. Yet no adequate explanation may be given to them if they ask. For instance, some patients might wish to point out that they can't remember whether or not there was any exception to nightmares on the Mondays, but such conversations are discouraged as per the M-FAST manual.

The M-FAST instructions are prone to make the PTSD patient feel rushed through the interview, not allowing them to properly comprehend the M-FAST questions and leaving them confused or mistrusting.

The patients' experience with the M-FAST is likely to produce data which are corrupt, with a significant bias toward detecting falsely high rates of malingering as well as carrying a decided risk of iatrogenic injury.

Of methodological interest is the manner in which the MFAST author, Holly A. Miller, described the "criterion validation" of her test, see pages 26-27 in the M-FAST manual [1]. A scientifically correct criterion validation is a demonstration that the test indeed does what it is purported to do [2], i.e., in the case of M-FAST, to differentiate between legitimate patients and malingerers. Instead, as a "criterion" validation, the M-FAST describes correlations to another test of malingering known as the Structured Interview of Reported Symptoms (SIRS) [6]: such correlations can be considered, at most, as indicative of so-called convergent validity, i.e., correlations with other measures of the same construct or with related clinical measures. The SIRS is not a flawless instrument providing an absolutely correct diagnostic classification. In fact, although substantially longer, the SIRS has similarities with the M-FAST. Some psychologists consider M-FAST as "essentially a short form of the SIRS" (see Graue et al. [7]). The similarity of SIRS and M-FAST unduly inflates the correlations of the two tests; their correlations in no manner demonstrate an adequate capacity of the M-FAST for differentiating malingerers from legitimate patients.

Years ago, when Miller "validated" her M-FAST [1], the SIRS (an early version of the currently used SIRS-2) was considered the "gold standard" for detection of malingering. According to a meta-analysis reported by Green and Rosenfeld,[8] research on the SIRS since its initial validation studies, however, has demonstrated lower specificity rates than those reported in the SIRS manual, i.e., a tendency towards higher rates of misclassifying patients as "malingerers," hence showing less ability to differentiate between the legitimate patients and malingerers.

Another alleged "criterion validity" study reported in the M-FAST manual (page 27) [1] is methodologically even less clinically sound. Miller compared M-FAST scores of students instructed to malinger mental illness with those responding honestly. This procedure differentiates only reporters from non-reporters of symptoms. Since the MFAST appears to mainly list legitimate medical symptoms [5], but scores them as indicative of malingering, Miller's "instructed malingerers" reported more of these symptoms than persons responding honestly. This method certainly does not verify the capacity of the M-FAST to differentiate malingerers from legitimate patients: both groups may report a similar number of symptoms.

This procedure of comparing scores of volunteers responding honestly to those of "instructed malingerers" is known as analogue validation [9]. A recent study 
demonstrated that any convenient list of legitimate medical symptoms, for instance, even the widely-used Beck Depression Inventory II (BDI-II) [10], could be "validated" via analogue validation procedures as a "test of malingering, with the disastrous result of hundreds of thousands of depressed patients being mislabeled as malingerers [9].

Experienced clinicians consider the M-FAST as mainly a list of legitimate medical symptoms [5], some of which could be legitimately endorsed by US combat veterans, depending on their current health condition.

The present study reviews tabular data published by Erika Wolf's team in 2020 on M-FAST responses of 121 US combat veterans with a probable current diagnosis of PTSD [3], [4]. The mean score of those with probable diagnosis of PTSD was $4.21(\mathrm{SD}=3.60)$ compared to only $1.93(\mathrm{SD}=1.73)$ of those without probable PTSD [3]. The difference is statistically significant, and thus documents that the M-FAST is more likely to misclassify patients with more severe PTSD symptoms as malingerers. Since the M-FAST has been shown to list mainly legitimate medical symptoms [5], it is important to examine which of its 25 items are endorsed by the veterans with the highest frequencies. We reviewed, from a clinical perspective, 7 M-FAST items that were endorsed by the highest proportions of these veterans.

\section{METHOD}

We used clinical perspective to explore and interpret the content of the most frequently endorsed M-FAST items by Wolf's 121 veterans with a probable current diagnosis of PTSD [3]. Using the highest items endorsement frequencies as our criterion, we re-arranged Wolf's published data [3] (as per Wolf's Table 2) to establish a rank order of the 25 items of the M-FAST and to identify the 7 most frequently endorsed items, see Table I. Such listing of most frequently endorsed items can provide an overall interpretable clinical profile of that group of veterans. Furthermore, we scrutinized the list of these most frequently endorsed items for their clinical potential of successfully differentiating malingerers from legitimate patients.

Our review comments only on 7 most frequently endorsed items, those when rounded reach each at least a $20 \%$ endorsement by the veterans. Items with lower frequencies risk to be clinically much less representative of those endorsed by other samples of veterans. In clinical psychology, e.g., in MMPI2 interpretations, psychologists also comment (with certain exceptions) only on several most elevated scales to establish the clinical profile of a group of patients in similar studies. The clinical profile thus consists only of the most salient and most likely replicable features.

An additional complication of selecting more than 7 items is that, in this sample of veterans, the next 3 items (those with next lower frequency) were all endorsed by the same proportion of veterans $(17.4 \%)$ : in statistical terms, thus representing "tied ranks," in addition to their presumably lesser relevance for establishing a replicable clinical profile of veterans.

Each M-FAST item, if endorsed in the direction considered by M-FAST system as "malingering," counts one point. The 7 items reviewed in our study, if all endorsed (as in the clinical profile on the M-FAST of this sample of veterans), would thus reach the official M-FAST cutoff score of 6 points. They represent items that most frequently misdiagnose legitimate patients as malingerers. The goal of the present study is to provide evidence, via accumulated clinical research and knowledge, of the fallacy of interpreting these legitimate symptoms, i.e., those most frequently endorsed by our veterans, as pathognomonic of malingering.

The wording of the M-FAST items is paraphrased here due to copyright protections as per the publisher of the M-FAST, but the emphasis is on conveying the clinical meaning of the items.

\section{RESULTS}

As explained, each M-FAST item, if endorsed in the alleged direction of malingering, counts one point, and the $\mathrm{M}$ FAST cutoff is 6 or more points.[1] Thus, our study focuses on 7 M-FAST items most frequently endorsed by the US combat veterans with probable PTSD, see Table I. These 7 items jointly represent the clinical profile of 121 US combat veterans, fallaciously considered in the M-FAST system as classifying this particular group as malingerers.

\begin{tabular}{lc} 
TABLE I: SEVEN MOST FREQUENTLY ENDORSED M-FAST ITEMS \\
\hline \multicolumn{1}{c}{ M-FAST item: } & \% Endorsement \\
\hline 2. Feeling depressed most of the time & $67.8 \%$ \\
23. I feel that I do not really matter & $56.2 \%$ \\
20. Often, I feel things crawling on me & $28.9 \%$ \\
when there is nothing there & \\
17. Smelling strange odors when I can't & $24.0 \%$ \\
fall asleep & \\
21. At times I hear music coming from \\
nowhere \\
$\begin{array}{l}\text { 14. As if someone were controlling my } \\
\text { symptoms, turning them on and off }\end{array}$ \\
\begin{tabular}{l} 
1. Restlessness in a chair \\
\hline
\end{tabular}
\end{tabular}

While we provide our clinical interpretations of this profile, the reader may wish to decide for himself or herself if any of these 7 most frequently endorsed items have any potential at all to successfully differentiate malingerers from legitimate PTSD patients. The following paragraphs review the item content from a clinical perspective, starting with items endorsed with the highest frequency.

\section{A. Endorsement of Item 2: Feeling Depressed All the Time}

About two-thirds of the combat veterans with a probable current diagnosis of PTSD endorsed "feeling depressed most of the time." Depression is certainly not uncommon among PTSD patients. Contributing factors in veterans could be events such as witnessing death of friends in combat or viewing wanton murders of civilians, including children. Depression is also a part of the lingering post-concussion syndrome sustained by many veterans by their repeated exposure to explosive blasts. The reader may recall that depression, in fact, is included as one of the clinically important items of the Rivermead Post-Concussion Symptoms Questionnaire [11], [12].

Even many lay persons recognize Item 2 as describing a legitimate medical condition, yet the M-FAST system scores this item falsely as an indicator of malingering. 


\section{B. Endorsement of Item 23: Most of the Time, I feel that I Do not Really Matter}

More than a half of combat veterans with probable current PTSD endorsed "feeling that I don't really matter." Unfortunately, such subjective impressions are reinforced by many veterans being falsely assessed as malingerers by the M-FAST or by similarly flawed tests such as the Structured Inventory of Malingered Symptomatology (SIMS) [13]-[20], or the Green's Medical Symptom Validity Tests (MSVT) [21], thus depriving them and their dependents of much needed therapies and benefits.

Rumours abound among veterans that some of their peers have been cheated during the compensation process. Those with low military rank might be especially prone to feeling that they do not matter and that they are treated accordingly.

\section{Endorsement of Item 20: I Feel Things Crawling on Me when Nothing is There}

Many M-FAST psychologists may be unaware that formication is a legitimate medical symptom. Formication is an abnormal sensation resembling that of insects crawling on the skin, in the skin, or under the skin [22]. In some cases, the sensation is associated with pain. The symptom is encountered in patients with various medical conditions, e.g., multiple sclerosis, exposure to environmental toxins, or cervical, thoracic, or lumbosacral whiplash (WAD-C, WADT, WAD-LS) [23]. A recent study of motorists injured in high impact motor vehicle collisions showed that $30.4 \%$ reported formication [22].

The endorsement of this item by $28.9 \%$ of combat veterans currently in the throes of PTSD suggests the possibility of injuries to afferent nerve fibers. This is likely from whiplash injuries sustained in explosive blasts during combat or from exposure to toxic substances in the war zone.

\section{Endorsement of Item 17: Smelling Strange Odors when I Cannot Fall Asleep}

Phantosmia is a false sensation of an odor, a phantom smell: $24.0 \%$ of combat veterans with probable current PTSD endorsed this item. This medical symptom occurs in Parkinson's disease [24], or as an olfactory hallucination in psychiatric patients, or may be experienced temporarily during migraines. It may also occur with mild traumatic brain injuries unassociated with visible external injury to the head. Phantosmia in veterans could represent a temporary or permanent sequelae of head trauma [25], [26] sustained in combat. The symptom may naturally appear more salient to the patient when not associated with usual daytime external distractions, e.g., while trying to fall asleep at night.

\section{E. Endorsement of Item 21: At Times I hear Music Coming from Nowhere}

Some musically inclined persons can replay music in their minds or hear music generated in their mind without making any conscious effort to recall its parts and without needing any external device to do so. Beethoven was known "to hear" music in his mind and was thus still able to compose entire symphonies after he lost his hearing completely. Thus, some normal persons could endorse this M-FAST item without any intent to malinger. Patients with intrusive mentation such as in PTSD are likely more prone to this phenomenon: $22.3 \%$ of veterans with probable current PTSD endorsed this M-FAST item.

F. Endorsement of Item 14: Sometimes it Seems as if Someone Were Controlling My Symptoms, Turning Them on and off

The symptoms of combat veterans with PTSD usually fluctuate in intensity over the days, weeks, and months. They may occur unpredictably on and off in distinct bouts. The patient might describe this unpredictable fluctuation of symptoms in words similar to "as though somebody controls my symptoms, turning them on and off ..."

In Wolf's study, $20.7 \%$ of the veterans with probable PTSD endorsed this M-FAST item. Wolf's research team subsequently estimated via interviews that in only $10.5 \%$ of these veterans such response to Item 14 might indicate malingering.

G. Endorsement of Item 1: Restlessness during the MFAST Administration (Observed versus Reported)

When the patient endorses Item 1 of the M-FAST indicating that he or she "often" finds it too difficult to sit still in a chair, the M-FAST manual instructs the examiner to observe carefully if this is indeed consistent with the nonverbal behavior of that patient. The discrepancy is scored as indicative of malingering. The scoring system of the MFAST ignores the possibility that the symptoms occur in bouts and independently of the M-FAST testing.

Restlessness is an important symptom of the postconcussion syndrome. It is listed as one of post-concussive symptoms in the widely used Rivermead Post-Concussion Symptoms Questionnaire [11], [12].

The semantic message of first words of Item 1 "I often find myself not being able to sit still ..." is open to alternative interpretations. In contrast to varied colloquial use of English, the M-FAST perhaps interprets the word "often," in this Item 1 , as synonymous with "always" or with "now, definitely during this interview." The bouts of restlessness, even the infrequent ones, can be perceived by the patient as subjectively aversive, annoying, or bothersome, and therefore as worth mentioning to an examiner who is expected to respond with empathy and explanation as well as offer to help, or at least a referral to another professional for the help.

\section{H. The Least Frequently Endorsed Items}

The least frequently endorsed items are those least likely characteristics for the clinical profile of a given group of patients. In Wolf's data, the least frequently endorsed by the veterans were M-FAST Items 6 (persistent hallucinations, endorsed by $1.7 \%$ ) and 13 (extraordinarily good eye sight, endorsed also by only $1.7 \%$ ). The endorsement of these items by $1.7 \%$ of the group (i.e., by 2 of the 121 veterans) could result from mishearing or misunderstandings (the patients are rushed through the M-FAST interview, responses to their questions are evasive, without clarification of item content, see instructions for administering the test in the M-FAST manual [1], page 10). However, other explanations might also seem plausible, such as impaired concentration, or symptoms of psychoses. 


\section{DISCUSSION}

Our article reviews the highest item frequency data that we have extracted from the psychometric study by Erica Wolf's team on 121 combat veterans with probable PTSD. Our goal has been to provide clinical perspectives on the most frequently endorsed M-FAST items because they build a clinical profile of this unique group of patients. Experienced clinicians are unlikely to consider any of the 7 items listed in our Table I as having a capacity to adequately differentiate between malingerers and legitimate patients. None of these most frequently endorsed items are pathognomonic, i.e., exclusive to malingering, but all 7 are erroneously scored by the M-FAST system as indicative of malingering. The following paragraphs sketch an overview of the most important methodological flaws of the M-FAST, relevant to this study.

\section{A. M-FAST's Systematic Bias towards False Positives}

As evidenced by their content, the 25 M-FAST items are an inappropriate tool to assess malingering in psychiatric patients, in persons injured in motor vehicle or industrial accidents, and in combat veterans [5]. The rates of false positives can be unacceptably high, when the test is used on real patients rather than in "simulation" studies on healthy college students. For example, a 2017 study by Weiss and Rosenfeld [27] on trauma-exposed African immigrants showed that the M-FAST "produced high false positive rates in the honest groups, ranging from $33 \%$ to $63 \%$."

Results of Wolf's 2020 study showed that all M-FAST items, without exception, show a systematic bias towards classifying the patient as a malingerer [3], [4]. On the basis of psychometric calculations, Wolf's VA team concluded "that despite its widespread use, the M-FAST may be insufficient for this purpose and that simply eliminating poorly performing items or conducting follow-up interviews may likewise be inadequate for addressing concerns related to the measure's reliability, validity, and utility in veteran PTSD samples.'[3].

\section{B. M-FAST's Fallacious Scoring of Legitimate Symptoms as Indicators of Malingering}

As explained, our study focused only on 7 most frequently endorsed M-FAST items by the 121 veterans in Wolf's study [3]. About two-thirds (67.8\%) of these veterans endorsed the M-FAST Item 2 ("feeling depressed most of the time") and more than a half $(56.2 \%$ ) endorsed Item 23 ("feeling that I don't really matter"). These two items denote depressive mood and discouragement: they fail to be pathognomonic of malingering or exclusive to malingerers.

Items 20 (formication, endorsed by 28.9\%) and 17 (phantosmia, endorsed by $24.0 \%$ ) are legitimate neuropsychological symptoms [22], [25], [26], potentially consistent with whiplash injuries or head trauma from repeated explosive blasts or with exposure to toxic chemicals [28] in combat. Subjective awareness of phantosmia or formication may be more salient when the patient is less distracted by external stimuli, e.g., while attempting to fall asleep (i.e., as specified by Item 17 "at times, when you cannot fall asleep"). Combat veterans as well as workers with head trauma from industrial accidents or injured motorists with the post-concussion and whiplash syndromes can all be misclassified by such M-FAST items that falsely score genuine neuropsychological impairments as indicators of malingering. Statistical analyses by Clark [29] have shown that the M-FAST is not an appropriate measure for identifying neurocognitive malingering.

Phantosmia is sometimes considered as synonymous with olfactory hallucinations. Perhaps it is preferable to consider phantosmia as a false olfactory sensation when it seems better explained by somatic factors such as head trauma or Parkinson's disease than by individual intrapsychic factors such as the patient's particular unconscious anxieties or an idiosyncratic psychotic mentation. However, there is no clear borderline between phantosmia seemingly related to a primarily somatic disorder and one with intrapsychic etiology as the two may be complementary or coexistent.

The next most frequently endorsed (by 22.3\%) was Item 21 ("At times I hear music coming from nowhere") which could represent, as suggested, spontaneous inner experiences of musically inclined individuals, albeit they also raise concern about their related propensity to intrusive thoughts as a manifestation of PTSD.

The perception that PTSD symptoms fluctuate unpredictably in frequency and severity may induce some veterans to endorse Item 14 ("Sometimes it seems as if someone were controlling my symptoms, turning them on and off," endorsed by $20.7 \%$ ).

The symptom of restlessness (Item 1) was endorsed by $19.8 \%$ of the veterans: it is more plausible to view it as a postconcussive symptom than a sign of malingering. A recent study on 89 athletes identified restlessness as a part of the "anxiety/mood subtype" of the post-concussion syndrome, together with irritability, depression, and impatience.[30] Further studies could clarify if this post-concussive subtype is related to generalized anxiety/agitation.

Briefly, the clinical profile of most salient symptoms of the US combat veterans may consist of depression, discouragement, neuropsychological signs of formication and phantosmia, an unpredictable fluctuation of PTSD symptoms, the restlessness, and perhaps also intrapsychic experiences of intrusive music melodies. Such a clinical profile is not pathognomonic of malingering. On the contrary, its main features are reasonably consistent with the clinical lore of post-traumatic symptoms prevalent in combat veterans. The 7 most frequently endorsed M-FAST items discussed here (endorsed by $19.8 \%$ to $67.8 \%$ of US veterans with a probable diagnosis of PTSD) illustrate the folly of the M-FAST scoring system.

\section{Fallacy of Observed Versus Reported Symptoms}

As explained, certain items require that the test administrator compare the patient's behavior during the interview to his or her M-FAST responses, for instance, the Item 1 (“.... often unable to sit still ...."). Thus, if the test administrator fails to observe nonverbal signs of restlessness during the interview, but Item 1 was endorsed, the M-FAST system scores this discrepancy as a sign of malingering. In everyday speech, the word "often" may be used as synonymous with "too often" to express personal displeasure with the subjectively aversive feeling of restlessness (as a post-concussive symptom), even if it is present only on some occasions and absent completely during the M-FAST 
interview. It also needs to be considered that the restlessness may be less intense while the patient is distracted by the demands and test tasks of the M-FAST interview than while at home.

\section{Ambiguity of Some M-FAST Items}

Semantically ambiguous items are especially likely to be misunderstood by patients whose concentration is impaired by insomnia (a symptom of PTSD or cerebral concussion, etc.) or by patients with thought disorder: the more severe such symptoms, the higher the M-FAST scores and rates of false positives. Briefly, patients who suffer more from their illness are most likely to be branded as malingerers by the MFAST. The test is clearly unsuitable for reliable forensic assessments of malingered severe mental illness by inmates of correctional facilities (the originally intended purpose of the M-FAST) or of malingered PTSD by combat veterans.

Wolf's team documented frequent mishearings or misunderstandings of M-FAST items by the veterans [3]. They are more likely when instructions for test administration in the M-FAST manual [1] (page 10) are followed blindly (the patient is rushed through the test, without proper pauses to think and to understand the items correctly, and questions are discouraged or responded to in an evasive style).

The scoring system of M-FAST seems to presume that patients are able to comprehend the logical structure of each item as do persons with the highest university education. As shown in the study by Wolf [3], even Item 5 ("mostly, I am feeling unusually happy"), formulated in simple English, was endorsed by some veterans who in subsequent clinical interviews explained that they assumed this question was about "feeling depressed." Perhaps their concentration was impaired by post-concussive symptoms, or some perceived the question about "unusual happiness" as a well-meaning sarcasm of an empathetic doctor. Item 5 was endorsed by $17.4 \%$ of veterans with PTSD during the M-FAST assessment, but the subsequent interviews suggested that only in $3.8 \%$ malingering may be involved.

Furthermore, the two-parts items of the M-FAST also contribute to misunderstandings when administered to patients with PTSD related insomnia or with post-concussive symptoms such as impaired concentration, or to those distracted by pain related fatigue.

Thus, even from the semantic perspective alone, the content of M-FAST items seems ill-equipped to differentiate malingerers from legitimate patients.

\section{E. Precarious Nature of Generalizations from "Instructed Malingerers"}

Using healthy volunteers instructed to malinger is a methodological weakness: they lack a true incentive. They usually are less well prepared to simulate the illness and are less cautious to avoid detection than genuine malingerers for whom much more is at stake. Cautious malingerers may spend time talking to real patients or perusing the library or internet for descriptions of the illness they plan to feign. For instance, healthy volunteers instructed to feign severe mental illness, but lacking in a genuine incentive to avoid detection, might obtain much higher M-FAST scores than do real malingerers and also than genuine severely ill psychiatric patients. In a study by Minoudis [31], the mean M-FAST score of instructed malingerers was $12.8(\mathrm{SD}=5.3)$ and the mean score of psychiatric patients was $5.2(\mathrm{SD}=5.7)$, i.e., more than twice higher.

As documented in various publications [14]-[20], the Structured Inventory of Malingered Symptomatology (SIMS) is also a list of legitimate medical symptoms falsely scored as indicative of malingering. Merten's imaginative German study [32] compared SIMS scores of two groups of volunteers instructed to malinger. The group of those warned to proceed cautiously to avoid detection obtained significantly lower SIMS scores than an unwarned group. Studies pretending to validate tests of malingering such as the M-FAST almost never test genuine patients against the genuine malingerers and hence such methodological designs produce crude overestimates of test validity, of its ability to detect true malingerers and separate them from legitimate patients.

\section{F. Fallacious Meta-analytic Studies of M-FAST by Detullio et al.}

Some readers may object to the thrust of the present article and point out that meta-analytic study by Detullio et al. [33] in 2019 statistically demonstrated an adequate specificity of the M-FAST, i.e., its capacity to avoid misdiagnosing legitimate patients. The so-called specificity statistics is particularly important in this context as it indicates the proportion of legitimate patients classified correctly as "nonmalingerers." Detullio's team claimed that their metaanalytically obtained average specificity for the M-FAST cutoff of 6 or more points was 0.85 . This would mean that only $15 \%$ of legitimate patients were incorrectly classified as malingerers.

Detullio's meta-analytic statistics [33] are fatally flawed due to methodological problems already discussed here, for example:

1) inclusion of "simulation studies," such as Miller's comparison of college students instructed to malinger with those instructed to respond honestly [1] (i.e., via methodologically illogical "analogue validation" [9] that even "successfully validated" BDI-II as a "test of malingering" [10]),

2) inclusion of other Miller's studies that used the SIRS (an imperfect tool, with noteworthy similarities to M-FAST) to identify "malingerers,"

3) and the confusion of statistics based on "instructed malingerers" with the test capacity to detect real malingerers.

Briefly, Detullio's meta-analytic estimates [33] of MFAST accuracy are crudely inflated by improper methodology.

\section{G. Veterans with Severe Mental Illness}

Severe mental illness such as schizophrenia can have its onset in the first decade of adult life, i.e., at the age when many of the veterans already became active members of the military. For example, a study on 112 Canadian patients shows that their first hospitalization for schizophrenia occurred at the mean age of 25.7 years $(\mathrm{SD}=7.5)$ [34]. A large proportion of M-FAST items describes mental health symptoms which can be endorsed by psychiatric patients with thought disorder, hallucinations, and delusions at rates indistinguishable from malingerers, [5] but these are scored, when endorsed, as indicative of malingering. Genuine, severely ill psychiatric patients are especially prone to misunderstand the text of some M-FAST items. This further 
inflates their "malingering scores." Such veterans with severe psychiatric symptoms are at high risk to be denied pharmacotherapies. Factors such as social rejection then cause many to become homeless.

\section{H. Position of the American Psychological Association (APA) on Clinical Tests}

The Standards stipulated by the APA for psychological tests [2] define test validity as "the degree to which all the accumulated evidence supports the intended interpretation of test scores for the proposed use." The M-FAST fails in this respect so extensively that it merits to be classified as "pseudopsychological test," for the same reasons as the Structured Inventory of Malingered Symptomatology (SIMS) [14]-[20].

According to APA Standards,[2] it is ultimately the responsibility of each individual test user to carefully evaluate the supportive and damaging evidence for the validity of the test, independently of claims made by the test publisher or test author. The APA Standards handbook [2] warns the test users: "Compliance or noncompliance with the Standards may be used as relevant evidence of legal liability in judicial and regulatory proceedings."

Many Canadian and US psychologists have concluded that M-FAST is a false and iatrogenic test. They are aware of its routine use by their peers and that no action has been taken to prevent this. Allowing peers to use the M-FAST on US combat veterans may deprive them of therapies or other benefits owed to them. Research by Murdock [35] has confirmed that "veterans who had been awarded PTSD service connection had clinically important reductions of PTSD symptoms, and reduced poverty and homelessness, compared with applicants whose claims had been denied," see comments in Marx et al. [36].

\section{CONCLUSIONS}

The 7 M-FAST items most frequently endorsed by US combat veterans with a probable diagnosis of PTSD provide a symptom profile that is consistent with the current state of clinical knowledge and research. From a clinical perspective, these 7 items obviously lack the capacity to differentiate malingerers from legitimate patients. Since more severely ill patients are likely to endorse more of such symptoms, they end up with higher "malingering" scores to their detriment.

\section{REFERENCES}

[1] Miller H. A. M-FAST. Miller Forensic Assessment of Symptoms Test. Professional Manual. Lutz, FL: PAR Inc., 2001.

[2] American Educational Research Association, American Psychological Association, National Council on Measurement in Education. Standards for Educational and Psychological Testing. Washington, DC: American Educational Research Association, 2014.

[3] Wolf E., Ellickson-Larew S., Guetta R. E., Escarfulleri S., Ryabchenko K., and Miller M. W. Psychometric performance of the Miller Forensic Assessment of Symptoms Test (M-FAST) in veteran PTSD assessment. Psychological Injury and Law. Published online April 15, 2020. https://doi.org/10.1007/s12207-020-09373-y.

[4] Wolf E. J., Ellickson-Larew S., Guetta R. E., Escarfulleri S., Ryabchenko K., Miller M. W. Correction to: Psychometric Performance of the Miller Forensic Assessment of Symptoms Test (MFAST) in Veteran PTSD Assessment. Psychological Injury and Law.
Published online: 02 September 2020. https://doi.org/10.1007/s12207020-09388-5.

[5] Cernovsky Z., Mann S., Diamond D. M., Persad E, Oyewumi L. K., Velamoor V., Mendonça J., Woodbury-Fariña M. A., and Husni M. Critical Review of the Content Validity of Miller Forensic Assessment of Symptoms Test (M-FAST). Archives of Psychiatry and Behavioral Sciences. 2020;3(2):16-29.

[6] Rogers R., Bagby R. M., \& Dickens S. E. SIRS: Structured Interview of Reported Symptoms: Professional Manual. Odessa, FL.: Psychological Assessment Resources, 1992.

[7] Graue L. O., Berry D. T. R., Clark J. A., Sollman M. J., Cardi M., Hopkins J., \& Werline D. Identification of Feigned Mental Retardation Using the New Generation of Malingering Detection Instruments: Preliminary Findings. The Clinical Neuropsychologist 2007;21(6):929-942. doi: 10.1080/13854040600932137.

[8] Green D., and Rosenfeld B. Evaluating the Gold Standard: A Review and Meta-Analysis of the Structured Interview of Reported Symptoms. Psychological Assessment. 2011, 23(1), 95-107.

[9] Cernovsky Z., Mann S., Diamond D. M., Mendonça J., Tenenbaum S., Persad E., Velamoor V., Woodbury-Fariña M. A., Husni M., Gutierrez J. Irremediably Flawed Nature of Analog Validation Methodology of Malingering Tests. Archives of Psychiatry and Behavioral Sciences. 2020; 3(2): 39-45.

[10] Beck A. T., Steer R. A., Brown G. K. Manual for Beck Depression Inventory-II. San Antonio: TX: Psychological Corporation; 1996.

[11] King N. S., Crawford S., Wenden F. J., Moss N. E. G., Wade D. T. The Rivermead Post Concussion Symptoms Questionnaire: a measure of symptoms commonly experienced after head injury and its reliability. Journal of Neurology. 1995;242:587-592.

[12] Cernovsky Z. Z., Mann S. C., Velamoor V., Oyewumi L. K., Diamond D. M., Litman L. C. Validation of the Rivermead Post-Concussion Symptoms Questionnaire (RPQ) on Patients Injured in High Impact Car Accidents. Archives of Psychiatry and Behavioral Sciences. 2021;4(1):14-22. doi.org/10.22259/2638-5201.0401003.

[13] Cernovsky Z. Z. Symptom profile of injured motorists on the Structured Inventory of Malingered Symptomatology. European Journal of Medical and Health Sciences. 2021;3(2):1-4. doi: 10.24018/ejmed.2021.3.2.739.

[14] Cernovsky Z., Mendonça J. D., Oyewumi L. K., Ferrari J. R., Sidhu G., and Campbell R. Content Validity of the Psychosis Subscale of the Structured Inventory of Malingered Symptomatology (SIMS). International Journal of Psychology and Cognitive Science. 2019;5(3):121-127.

[15] Cernovsky Z. Z., Mendonça J. D., Ferrari J. R., Sidhu G., Velamoor V., Mann S. C., Oyewumi L. K., Persad E., Campbell R., and WoodburyFariña M. A. Content Validity of the Affective Disorder Subscale of the SIMS. Archives of Psychiatry and Behavioral Sciences. 2019;2(2):33-39.

[16] Cernovsky Z., Bureau Y., Mendonça J., Varadaraj Velamoor V., Mann S., Sidhu G., Diamond D. M., Campbell R., Persad E., Oyewumi L. K., and Woodbury-Fariña M. A. Validity of the SIMS Scales of Neurologic Impairment and Amnestic Disorder. International Journal of Psychiatry Sciences. 2019;1(1):13-19.

[17] Cernovsky Z. Z., Mendonça J. D., Ferrari J. R., Bureau Y. R. J. Content validity of SIMS low intelligence scale. International Journal of Research in Medical Science. 2019;1(1):21-25.

[18] Widows M. R., Smith G. P. Structured Inventory of Malingered Symptomatology - Professional Manual. Lutz, FL: PAR Inc., 2005.

[19] Cernovsky Z. Z., Mendonça J. D., Ferrari J. R. Meta-Analysis of SIMS Scores of Survivors of Car Accidents and of Instructed Malingerers. Archives of Psychiatry and Behavioral Sciences. 2020;3(1):1-11.

[20] Cernovsky Z. Z., Ferrari J. J. R., Mendonça J. D. Pseudodiagnoses of Malingering of Neuropsychological Symptoms in Survivors of Car Accidents by the Structured Inventory of Malingered Symptomatology. Archives of Psychiatry and Behavioral Sciences. 2019; 2(1): 55-65.

[21] Ferrari J. J. R., Cernovsky Z. Z., Mendonça J. D. False Positives in Green's Tests of Malingering on Chronic Pain Patients. International Journal of Psychology and Cognitive Science, 2019; 5(2): 58-63.

[22] Cernovsky Z. Z., Velamoor V. R., Mann S. C., Oyewumi L. K., Mendonça J. D., and Litman L. C. Frequency of Formication Symptoms in Injured Motorists and in Normal Controls. European Journal of Medical and Health Sciences. 2021;3(2):48-52.

[23] Cernovsky Z. Z., Mann S. C., Velamoor V. R., and Oyewumi L. K. The Need for Three Separate Parallel WAD Ratings of Whiplash Injuries to Cervical, Lumbosacral, and Thoracic Spine in Clinical Assessments of Injured Motorists. European Journal of Medical and Health Sciences. 2021;3(1):154-159. doi: 10.24018/ejmed.2021.3.1.699.

[24] Landis B. N. and Burkhard P. R. Phantosmias and Parkinson Disease. Archives of Neurology. 2008; 65(9):1237-1239. 
[25] Lötsch J., Ultsch A., Eckhardt M., Huart C., Rombaux P., and Hummel T. Brain lesion-pattern analysis in patients with olfactory dysfunctions following head trauma. Neuroimage: Clinical. 2016; 11: 99-105. doi: 10.1016/j.nicl.2016.01.011.

[26] Doty R. L., Yousem D. M., Pham L. T., Kreshak A. A., Geckle R., Lee W. W. Olfactory Dysfunction in Patients with Head Trauma. Archives of Neurology. 1997 54(9):1131-40. doi: 10.1001/archneur.1997.00550210061014.

[27] Weiss R. A. and Rosenfeld B. Identifying Feigning in Trauma-Exposed African Immigrants. Psychological Assessment. 2017; 29(7):881-889.

[28] Thawani S., Wang B., Shao Y., Reibman J. and Marmor M. Time to Onset of Paresthesia Among Community Members Exposed to the World Trade Center Disaster. International Journal of Environmental Research and Public Health. 2019, 16, 1429.

[29] Clark J. A. Validation of the Miller Forensic Assessment of Symptoms Test (M-FAST) in a civil forensic population. Master's Thesis, University of Kentucky, 2006.

[30] Maruta J., Lumba-Brown A., and Ghajar J. Concussion Subtype Identification with the Rivermead Post-concussion Symptoms Questionnaire. Frontiers in Neurology. 2018;9:1034. doi: 10.3389/fneur.2018.01034.

[31] Minoudis P. G. Malingering of Cognitive Symptoms. Doctoral Thesis, University College London, London, UK, 2007.

[32] Merten T., Diederich C., Stevens A. Feigned Symptoms after Whiplash Injury: An Experimental Analogue Study. Aktuelle Neurologie. 2008;35:8-15. doi.org/10.1055/s-2007-986232.

[33] Detullio D., Messer S. C., Kennedy T. D., and Millen D. H. A MetaAnalysis of the Miller Forensic Assessment of Symptoms. Test (MFAST). Psychological Assessment. 2019;31(11):1319-1328. 10403590/19/\$12.00 http://dx.doi.org/10.1037/pas0000753.

[34] Cernovsky Z. Z., Landmark J. A., O’Reilly R. L. Age of onset and symptom patterns in schizophrenia. European Journal of Psychiatry. 2002; 16(3): 168-173.

[35] Murdoch M., Sayer N. A., Spoont M. R., Rosenheck R., Noorbaloochi S., Griffin J. M., et al. Long-term outcomes of disability benefits in US veterans with posttraumatic stress disorder. Archives of General Psychiatry.

2011;68:1072-1080. doi:10.1001/archgenpsychiatry.2011.105

[36] Marx B. P., Jackson J. C., Schnurr P. P., Murdoch M., Sayer N. A., Keane T. M., Friedman M. J., Greevy R. A., Owen R. R., Sinnott P. L., and Speroff T. The reality of malingered PTSD among veterans: Reply to McNally and Frueh (2012). Journal of Traumatic Stress. 2012;25:457-460. https://doi.org/10.1002/jts.21714.

Zack Z. Cernovsky was born in 1947 and holds the Ph.D. in clinical psychology from the University of Zürich, Switzerland, 1986. He is the professor of psychiatry in the medical school of Western University, Canada. Professor Cernovsky has published more than 200 scientific articles in the field of psychiatry and medical psychology, and also chapters in university textbooks.

Stephan C. Mann was born on May 6, 1948 in Philadelphia, Pennsylvania, USA. He received his medical degree from the Sidney Kimmel Medical College of Thomas Jefferson University where he also completed his residency in psychiatry. He worked for the United States Veterans Health Administration for over thirty years and retired as Chief of Mental Health \& Behavioral Sciences at the Louisville VA Medical Center, Louisville, KY. He is currently in private practice. Dr. Mann has previously held faculty positions as Professor of Psychiatry at both the University of Pennsylvania School of Medicine and the University of Louisville School of Medicine. He is a Distinguished Life Fellow of the American Psychiatric Association and is certified by the American Board of Psychiatry and Neurology. He has published almost one hundred scientific publications including three books

Dr. Mann's main research focus includes neuroleptic malignant syndrome, malignant catatonia, tardive dyskinesia, and the neurobiology of schizophrenia.

Varadaraj R. Velamoor graduated in Medicine from Osmania University in India and received his training in Psychiatry in the United Kingdom. He is currently Professor of Psychiatry at the Northern Ontario School of Medicine as well as Professor Emeritus at the Western University School of Medicine. He was previously on Faculty at Cornell University. Professor Velamoor has published over a hundred scientific articles as well as book chapters and psychiatric monographs in the areas of suicidal behaviour, violence, stress at the workplace, collaborative care, geriatric psychopharmacology and the Neuroleptic Malignant Syndrome (NMS). His work in NMS is internationally recognized and frequently cited. He has received Fellowships from the Royal College of Psychiatrists, UK, as well as the American Psychiatric Association. 